\title{
Lightweight multi-agent framework for a cluster-based wireless sensor network
}

\author{
Shahzadi Bano \\ IICT MUET \\ Jamshoro, Pakistan \\ szghunio@gmail.com
}

\author{
Syeda A. Afghan \\ Faculty of Informatics \\ University of Debrecen \\ Hungary \\ adila@eng.unideb.hu
}

\author{
Sana H. Jokhio \\ Melbourne, Australia \\ shoorjoks@gmail.com
}

\author{
Shahnawaz Talpur \\ Dept. of CSE, MUET \\ Jamshoro, Pakistan \\ shahnawaz.talpur \\ @ faculty.muet.edu.pk
}

\author{
Imran A. Jokhio School \\ of IT \& Eng. MIT \\ Melbourne, Australia \\ ijokhio@mit.edu.au
}

\begin{abstract}
Sensor applications and wireless sensor networks (WSNs) are becoming a part of our everyday life. A number of network arrangements are used in WSN. In this paper, we focus on the cluster based network to help identify the issues associated with communication within such networks. We present a lightweight multi-agent routing framework for a cluster based WSN to resolve some issues associated with such networks. By using state- of-art protocol in a unique combination and categorizing cluster layers, we take full advantage of the properties of the selected protocols. The simulation results illustrate that the proposed method is light-weight in terms of energy consumption by the sensor nodes communicating information within a cluster based network. Nevertheless, high network throughput and robust data communication are also achieved.
\end{abstract}

Index Terms-WSN, cluster based routing, light-weight

\section{INTRODUCTION}

WSN are self-configuring and are deployed to sense and monitor physical phenomena e.g. environment. A major limitation of WSN is that the nodes are battery-operated nodes. The sensor uses short-range wireless communication that may require mobility of the node and has a lower degree of central management and limited memory. All these constraints may affect the communication of data in a WSN [1]. Wireless Sensor Networks (WSNs) is an important revolutionary change in technology with a great potential for improving many current applications and are befitting in various areas including habitat monitoring, building surveillance, forest surveillance, earthquake observation, etc. These application are physically environmental but now a days these applications play a vital role in biological science, biomedical and health care, vehicle tracking. In these applications sensors are frequently scattered remotely and are made to operate independently autonomous [1]-[3]. At times the conditions are on extremity, but these nodes collect the data, process the data and now this data is ready for boost up that processed data towards the base station with assistance of intermediate cluster heads or adjacent nodes with the single hope or multiple hope communication [4], [5]. There are many significant events during the communication i.e.

- how to collect data

- which data is need to be dispensed

- how cluster is arranged in the design

- how to confine cluster boundaries
- how data is agglomerate at the cluster head

- how data is move to the base station for further processing

- how to fortify this data at per unit step [6] etc.

Lifetime of a network and ability to serve all the services to the application are most assertive parameters, well thought out in designing the protocol for WSNs.

Absorbing low power and proliferation of network life time are two important attributes of any routing protocol for WSNs. i.e. the protocol should guarantee that connectivity in the network is maintained for large duration and the energy rating of the entire network should be systematize in the same way. To yield a long duration of connectivity from center is only possible if there is an equal distribution of energy within the nodes of network [7]-[9]. Nowadays a variety of applications are being used in those fields where access is very difficult like battle field surveillance, whether monitoring, disaster man- agement, petroleum, controlling and sensing environment; use wireless sensor networks. Each wireless sensor is a countered with tiny sensing devices, wireless communication, finite radio range and band width, each node is capable to process, collect and transmit data to any one of the destined base station [10]. By the all of three drawbacks the top leading challenge is energy consumption and extending network life time specifi- cally for sensor deployed in the surrounding that are not easily accessible. As a consequence it is infeasible to substitute the sensor battery. So it is essential for WSNs to efficiently utilize energy and abate cost [11]-[13].

The applications of WSNs are increasing day by day. Vari- ous types of network arrangements are used in WSN [14]. We focus on multi-level cluster based network architecture. It may not be a good idea to use a single type of routing mechanism at all levels of communication in a cluster-based network. Nevertheless, keeping the WSN constraints in mind, the overall routing solution should focus on energy consumption, device type, etc. to guarantee communication at multi-layer [15]. Our research will highlight the routing challenges in a multi-layer WSN architecture and provide a lightweight multi- agent framework for efficient routing solution (combination of various state-of-art protocols) for it by considering the WSN constraints.

The rest of the paper has been organized as follows. Section 2 discusses the literature review in detail and some 
related work has also been presented here. Section 3 covers details regarding our proposed solution to the routing issues in cluster based WSN, its design, development, implementation, simulation setup and criteria, etc. Section 4 is dedicated to the detailed implementation setup and discusses the tools and technologies which are used to implement the proposed solution. Nevertheless, testing and analysis phases have also been discussed in this section. Moreover, a detailed discussion regarding the obtained simulation results have also been provided here. We conclude our findings in Section 5. Some future research directions have also been discussed here.

\section{RELATED WORK}

A routing mechanism is necessary in a network to manage the communication and information sharing among nodes and the base station. In a WSN, routing plays an important role due to its constraints and the nature of the application. The WSN constraints such as limited battery, limited processing power, limited bandwidth, etc makes a WSNs routing protocol design a challenging task. One of the major issues focused is the network life time. The energy consumption by a sensor node needs to be as little as possible in order to avoid node failure. Nevertheless, it is not possible to change the batteries of the sensor nodes frequently (low maintenance) hence if one node saves energy, gradually saving energy of more nodes associated with the network may result in an overall increase in the lifetime of the network. A number of routing protocols have been proposed globally trying to address the issues related to the WSN communication. A few are discussed below.

M. J. Handy, et al. proposed LEACH; a Low-Energy Adaptive Clustering Hierarchy protocol [17] which is one of the routing protocols that focused hierarchical approach for sensor network routing. LEACH protocol is adaptive and selforganizing in nature and claimed to have reduced the energy consumption by the sensor nodes significantly. Scalability, dynamic clustering, single-hop communication, etc., are a few pros to name. However, the working involves cluster heads being responsible for the data transmission which results in more energy consumption by the cluster heads (CHs). This may result in shorter life time of the $\mathrm{CH}$. Nevertheless, if a $\mathrm{CH}$ dies, it may result in the entire cluster being cut off from the network as no alternative means of communication is possible. All the end nodes only communicate with the cluster head. The $\mathrm{CH}$ further transmits information to the base station (BS). Moreover, the cluster distribution is non-uniform which results in $\mathrm{CH}$ being located at edges of a cluster. This also may result in an increase in the energy consumption as the $\mathrm{CH}$ may be located at greater distance from the BS hence more power consumed to transmit information if the network is distributed over a large geographical area. Random selection of $\mathrm{CH}$ also makes it difficult to confirm the total number of cluster heads in a network and their distribution.

W.B. Heinzelman et al. in [18] proposed LEACH-C, a centralized version of $\mathrm{LEACH}$ protocol. It is an extension to the LEACH protocol that includes a centralized clustering algorithm that involves base station for cluster formation. Hence it does not have the self-organizing nature as LEACH. It is claimed to reduce the energy consumption via selecting the $\mathrm{CH}$ based on the location information and the member nodes. Localization of the sensor nodes requires more energy hence LEACH-C may not always be a better alternative than LEACH protocol itself. The reason that it is able to transmit more data than LEACH makes it favorable and efficient.

S. Lindsy and R. Raaghavendra presented a PowerEfficient Gathering in Sensor Information Systems (PEGASIS) in [19] a chain-based protocol in nature. This protocol also is an extension of the LEACH protocol. The communication in PEGASIS is based on neighbor talk. Each sensor node only communicates with its neighbor to send and receive the data. The total throughput is increased by implementing PEGASIS protocol in other words the overall performance is doubled as compared to that of $\mathrm{LEACH}$ protocol. However, the protocol faces a major issue that is redundant data transmission.

O. Younis and S. Fahmy in [22] proposed HEED; a Hybrid Energy Efficient Distributed Clustering protocol. It is based on multi-hop communication and includes energy distribution algorithm. Sensor location information is not needed for communication of information. However, it carries out random selection of the $\mathrm{CH}$ from time to time hence this might give rise to the energy consumption of the sensor nodes due to cluster reconstruction.

Manjeshwar and D. Agrawal presented Threshold sensitive Energy Efficient sensor Network protocol (TEEN) [20]. This protocol is designed for WSN applications that sense sudden or abrupt changes or phenomenon. It is a hierarchical protocol. The network may operate in a reactive mode in such applications. Such kinds of applications are sensitive in nature and are responsible for time-critical decisions. The pros include better throughput. However, the desired performance is not obtained where the WSN covers a large geographical region. More energy is also consumed due to long distance transmission of information in such condition.

Q. Li, et al. [21] proposed a Hierarchical Power-Aware Routing (HPAR) scheme. This scheme categorizes the sensor nodes in a WSN into zones. Each zone consists of a group of sensor nodes that are geographically located close to each other or in other words, are neighbors. This group is known as an entity. Once the zones are formed the scheme then selects the routing paths across other zones that guarantee an increase in the sensor nodes battery life. This is done in a hierarchical manner. This scheme considers the nodes battery power that is involved in the communication or is on the routing path. Nevertheless, the transmission power is also considered. The zones on the other hand involve a large number of nodes hence overall periodic battery life estimations may cost more energy and burden the network with excess communication.

A number of solutions have been proposed globally. All have their own unique way of solving the routing challenges in a cluster based network. However, if considered, different layers of a cluster based network have different requirements and issues. One protocol might work better on lower layer 
and not so well on the higher layers. This may be due to configuration of nodes, communication overhead, large geographical networks, etc. If carefully evaluated separate suitable protocols may be selected to route information on different layers in a cluster based network. We discuss our proposed method in the next selection.

\section{LIGHT-WEIGHT MULTI-AGENT ROUTING FRAMEWORK}

This section discusses the proposed framework and its design. A detailed discussion regarding the choice of the stateof-art protocols for the framework is also presented here. In a cluster based network, the end nodes communicate with the cluster heads and cluster heads are responsible for transmitting the information regarding the reported phenomenon by the end nodes to the base station. Hence, using single routing protocol at all the cluster layers might not prove to be efficient enough to help achieve a better network lifetime. The WSN constraints such as batterys life, communication overhead, etc should be considered to help increase the overall efficiency and lifetime of the network. In this proposed framework we consider two scenarios; single agent and multi-agent. Two state-of-art protocols are chosen that are implemented using two different scenarios to evaluate their properties. The two scenarios are discussed below in detail.

\section{A. Scenario 1}

A cluster based WSN with a single routing algorithm responsible for communication within the network; end node to cluster head and to base station, is considered in this scenario. Hence, this scenario actually helps evaluate the pros and cons of the state-of-art routing protocol with respect to cluster based WSN. The main issues faced while using a single agent are:

- Communication overhead

- Networks lifetime may decrease

- Energy consumption by end nodes and the cluster heads

- Bottlenecks due to change in demand of communication within various types of nodes.

Both protocols are implemented individually and independently over a WSN. The communication is considered among end node to cluster head and among cluster head to base station (Figure 1). Hence, two layers are considered in a cluster based network. Various network sizes are also considered to evaluate the protocols with respect to scalability.

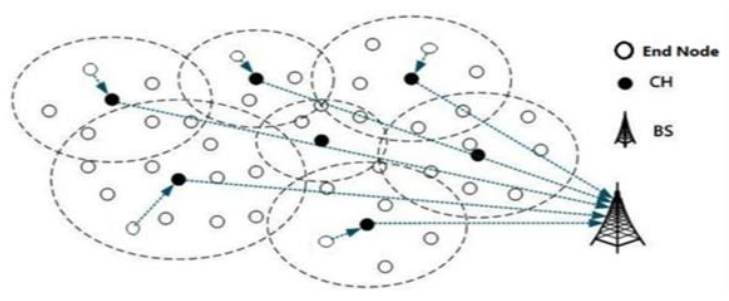

Fig. 1. Scenario 1 - Single Agent

\section{B. Scenario 2}

In this scenario, the two protocols are implemented on different network layers assuming they are best suited at that layer with respect to their claimed properties. Protocol 1 is responsible for the communication between end nodes and a cluster head whereas, protocol 2 is used for the communication among the cluster heads and base station. This protocol is also responsible for the communication among cluster heads. The figure 2 below illustrates the idea. For the proposed

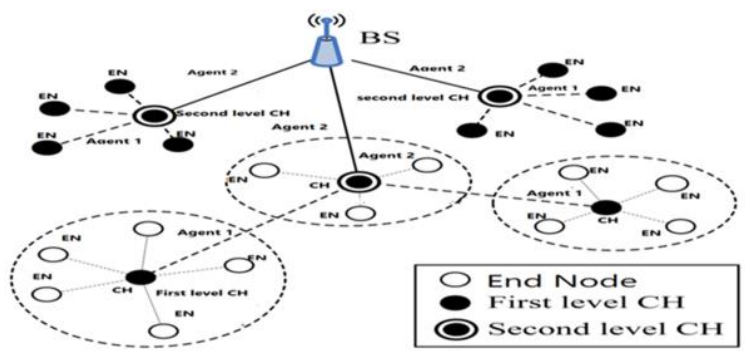

Fig. 2. Scenario 2 - Multi Agent

framework, two protocols are selected, Low Energy Adaptive Clustering Hierarchy (LEACH) and Power-Efficient Gathering in Sensor Information System (PEGASIS). These protocols are claimed to have been working on cluster based network with good efficiency. A brief discussion regarding their properties and lacks is given below.

\section{Agents}

LEACH and PEGASIS are claimed to have properties such as they provide extended lifetime to the network and energy consumption is quite low as compared to other state-of-art pro- tocols. Both these properties are promising hence are evaluated using the two proposed scenarios. LEACH protocol divides or arranges the sensor nodes in a WSN into small clusters. One of the nodes is then selected as a cluster head $(\mathrm{CH})$. The sensor node, also known as an end node, detects a phenomenon and reports it to the cluster head of the cluster it is in. The cluster head, after receiving the information from all nodes in a cluster, compresses it and sends it to the base station. The cluster head nodes consume more energy as compared to the end nodes within the cluster while communicating the information as the base station might be distantly physically located.

The cluster heads on the network are randomly selected to equalize the energy consumption by nodes within a network. Hence not all the nodes get a chance of being a cluster head in the network. This protocol may work efficiently where the sensor nodes need to constantly monitor certain activities within the network. The advantages of LEACH include:

- Single hop communication helps reduce energy consumption (end nodes to cluster head or cluster head to base station)

- The cluster head is responsible for collection of data which results in a decrease in overall network traffic 
- The overall communication is less hence the sensor node energy consumption is less

- Sensor location is not required for communication and formation of a cluster

- The distributive nature of LEACH makes it favorable

However, some drawbacks are also associated with LEACH protocol such the number of cluster heads on a network cannot be known. One of the major issues with LEACH is with respect to the cluster heads life time as there is no alternative to provide information to the base station regarding any faults at the cluster head. Moreover, random distribution and cluster formation does not equally arrange the end nodes hence may result in some busy clusters. Nevertheless, some cluster heads may be located at the center whereas others at the edges, resulting in more energy consumption by the cluster head to communicate and receive information.

Some of the issues in LEACH, if used in combination with another protocol, may be resolved. We have chosen PEGASIS (Power-Efficient Gathering in Sensor Information Systems) as our second protocol to work in a multi layer cluster based network environment.

It has been proposed as an extension to LEACH protocol and claims to have been more energy efficient than LEACH. The nodes that are located far from the base station use up more energy in communication as compared to those located close to it. Hence, the idea of transmitting the information directly by end nodes to the base station might not be feasible based on the location of the node. The energy may also be saved by making some sensor nodes in a cluster as member nodes. These member nodes may sense and compute the data which is known as data fusion. Therefore, the cluster head only transmits the fused data to the base station hence reducing the processing overhead on it. This helps reduce the energy consumption by the cluster head. Moreover, all sensor nodes can take turns to become a cluster head of a cluster or become a member. Hence the overall energy consumption by the sensor nodes in a cluster is reduced. PEGASIS help transmit information to the nearest neighbor sensor nodes to save energy. All the nodes receive data from neighboring nodes, fuse it and pass it on to the next neighbor until the data reaches the base station. Here every sensor node becomes a leader at some point in time and is responsible for transmitting the fused data collected from the nodes to the base station. However, there are some disadvantages associated with PEGASIS:

- The energy consumption is still high

- Delays are associated with the communication as all the nodes are involved in collecting and fusing data

- Proper topology is needed to be able to collect and communicate the data

- In case of a node failure during data fusion, the source to destination chain needs to be constructed again.

Although there are advantages and disadvantages associated with both the protocols but we believe that if used on different layers of the cluster based WSN, the overall network efficiency, life time, robustness in communication may be achieved. We discuss simulation and results in the next section.

\section{SIMULATION AND RESULTS}

The two scenarios discussed in the previous section are simulated and tested using Network Simulator (NS) [16]. The total number of sensor nodes used for the simulation

100. Sub-scenarios were simulated to test for the complexity of the sensor node deployment and communication. These scenarios contained 20,40,60,80 and 100 sensor nodes respectively. For Scenario 1, the protocols were simulated and tested individually and the results were obtained with respect to throughput, energy consumption and delay. Whereas, for Scenario 2, the two protocols were simulated using a cluster based network model in which the two protocols worked on different cluster levels. The graphical representation of the simulation results is presented, analyzed and discussed below. For performance analysis, different combination of network complexities is used. Throughput of all routing protocols (with respect to Scenario 1 and 2) is shown in figure 3. With increasing complexity, throughput is affected for LEACH and PEGASIS whereas the proposed combination works better while increasing the number of nodes in the network model. LEACH protocol seems to have worked better than PEGASIS but still it has lower throughput with respect to network size than the proposed combination of the protocols. The proposed combination has better performance as it actually uses the properties of the two protocols in combination. As network complexity increases as results of combine protocol rises more due to more paths to deliver more amount of data.

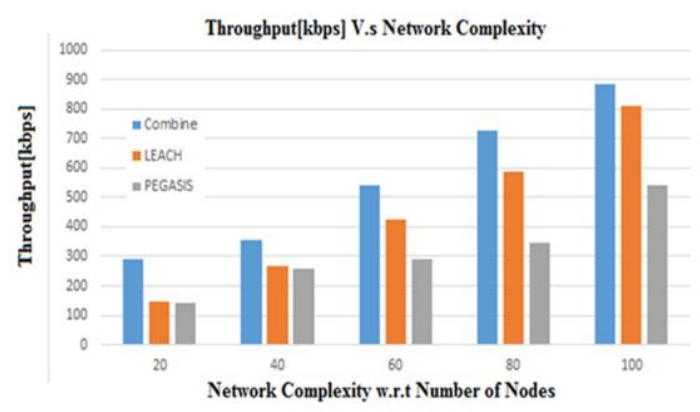

Fig. 3. Throughput

Figure 4 shows delay in the communication in the network. The actual delay depicts the time a packet takes to reach its destination. This also indicates the overall or total traversal time of the packet. PEGASIS has more delays with increasing complexity of the network as compared to the other two scenarios where LEACH protocol works independently and later with PEGASIS itself. When working together, the protocols seem to have overcome the delay issues in network communication while increasing the number of nodes or the network size. However, LEACH protocol while working independently also tackles the delay issues to some extent and has better output as compared to the PEGASIS protocol. 


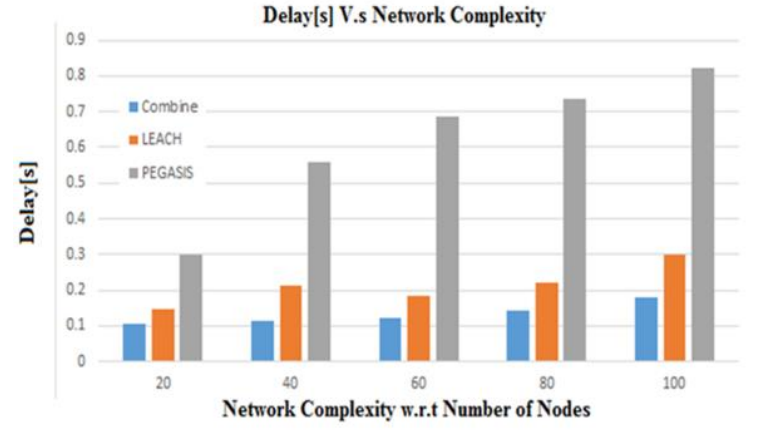

Fig. 4. Delay

Energy consumption plays a vital role in maintaining a networks lifetime. Figure 5 illustrated the comparison of total energy consumed by network nodes while using the three different protocol setups. Network consumes more energy as its complexity increases. As the number of nodes increase the communication among the nodes and to the base station also increases resulting in more energy consumption by the sensor nodes involved in the communication. PEGASIS seem to be less energy efficient as compared to the other two setups and hence has more energy consumption as the number of nodes increase in the network. However, LEACH protocol and the proposed combination perform better

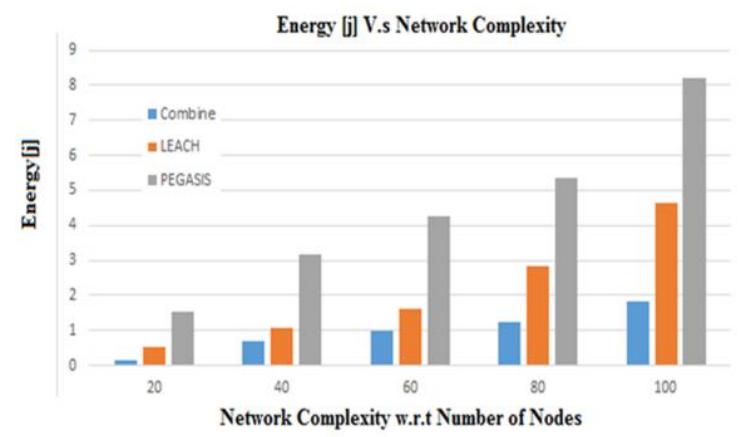

Fig. 5. Energy Consumption

The overall results show that the PEGASIS protocol does not provide efficient results with respect to increasing the number of nodes in the network while working independently. However, LEACH protocol, to some extent, works better independently and also works fine with increasing network size. Whereas, our proposed combination of the two takes full advantage of the properties of the two protocols and seem to provide efficient and robust solution for the cluster based network. Not only it shows that it supports scalability, but it also provides energy efficient robust communication method for a multi-layered cluster based network.

\section{CONCLUSION}

One of the major challenges in a protocol design for a WSN is network lifetime. In this paper, we proposed a lightweight multi-agent framework for cluster based WSN that considers the WSN constraints to improve the overall performance of the network. This framework actually is a unique combination of two protocols, PEGASIS and LEACH that are used to work together in a multi-layer cluster based network. The proposed combination was simulated and compared with respect to the chosen protocols working independently on a cluster based network. The simulation results showed that the proposed combination worked better as compared to the protocols work- ing independently on a cluster based network. The proposed method showed good network throughput, energy efficiency and less communication delay. Nevertheless, it supported network scalability and help increase overall network lifetime for a multi-layer cluster based wireless sensor network.

\section{REFERENCES}

1. [1] Li, C.; Zhang, H.X.; Hao, B.B.; Li, J.D. A survey on routing protocols for large-scale wireless sensor networks. Sensors 2011, 11,34983526 .

2. [2] Prasath, K.A.; Shankar, T., "RMCHS: Ridge method based cluster head selection for energy efficient clustering hierarchy protocol in WSN," in Smart Technologies and Management for Computing, Communication, Controls, Energy and Materials (ICSTM), 2015 International Conference on , vol., no., pp.64-70, 68 May 2015.

3. [3] Wei, C.; Yang, J.; Gao, Y.; Zhang, Z. Cluster-Based Routing Protocols in Wireless Sensor Networks: A Survey. In Proceedings of 2011 In- ternational Conference on Computer Science and Network Technology, Harbin, China, 2426 December 2011; pp. 16591663

4. [4] Abdullah, J.; Zeni, S., "Maximizing the network lifetime of clustered- based WSN using probability of residual energy," in Control System, Computing and Engineering (ICCSCE), 2014 IEEE International Con- ference on , vol., no., pp.178-183, 28-30 Nov. 2014.

5. [5] Xu, D.; Gao, J. Comparison study to hierarchical routing protocols in wireless sensor networks. Procedia Environ. Sci. 2011, 10,595600 .

6. [6] Roslin, S.E., "Genetic algorithm based cluster head optimization using topology control for hazardous environment using WSN," in Innovations in Information, Embedded and Communication Systems (ICIIECS),

7. 2015 International Conference on , vol., no., pp.1-7, 19-20 March 2015.

8. [7] Roy, S.; Kumar Das, A., "Energy efficient cluster based routing pro- tocol (EECBRP) for Wireless Sensor Network," in Networks and Soft Computing (ICNSC), 2014 First International Conference on, vol., no., pp.25-29, 19-20 Aug. 2014.

9. [8] Chakraborty, S.; Khan, A.K., "A noble approach for self learning and cluster based routing protocol with power efficiency in WSN," in Communications and Signal Processing (ICCSP), 2014 International Conference on , vol., no., pp.773-777, 3-5 April 2014.

10. [9] Haneef, M.; Deng, Z. Design challenges and comparative analysis of cluster based routing protocols used in wireless sensor networks for improving network life time. Adv. Inf. Sci. Serv. Sci. 2012, 4, 450459.

11. [10] Ishmanov, F.; Malik, A.S.; Kim, S.W. Energy consumption balancing (ECB) issues and mechanisms in wireless sensor networks (WSNs): A comprehensive overview. Eur. Trans. Telecommun. 2011, 22, 151167.

12. [11] Gemeda, K.A.; Gianini, G.; Libsie, M., "The effect of node selfishness on the performance of WSN cluster-based routing algorithms," in AFRICON, 2015 , vol., no., pp.1-5, 14-17 Sept. 2015. 
13. [12] Abdulsalam, H.M.; Kamel, L.K. W-LEACH: Weighted Low Energy Adaptive Clustering Hierarchy aggregation algorithm for data streams in wireless sensor networks. In Proceedings of IEEE International Conference on Data Mining Workshops (ICDMW), Sydney, Australia,

14. 14 December 2010, pp. 18.

15. [13] Zeb, A.; Islam, A.K.M.M.; Komaki, S.; Baharun, S., "Multinodes join- ing for dynamic cluster-based Wireless Sensor Network," in Informatics, Electronics and Vision (ICIEV), 2014 International Conference on , vol., no., pp.1-6, 23-24 May 2014.

16. [14] Hong, J.; Kook, J.; Lee, S.; Kwon, D.; Yi, S. T-LEACH: The method of threshold-based cluster head replacement for wireless sensor networks. Inf. Syst. Front. 2009, 11, 513521 
17. [15] Loscri, V. ; Morabito, G. ; Marano, S., A Two-Levels Hierarchy for Low-Energy Adaptive Clustering Hierarchy (TL-LEACH), Vehicular Technology Conference, 2005. VTC-2005-Fall. 2005 IEEE 62nd, pp

18.

19. [16] Network simulator 2 (NS2), www.isi.edu/nsnam/ns/ [Last accessed 26-

20. 12-2017].

21. [17] Handy M. J.; Haase M.; Timmermann D.; Low Energy Adaptive Clus- tering Hierarchy with Deterministic Cluster-Head Selection, In Proc.4th International Workshop on Mobile and Wireless CommunicationsNet- work, USA, 2002, Vol. 1, pp. 368-372.

22. [18] Heinzelman W. B.; Chandrakasan A. P.; Member S. and Balakrishnan H., An Application-Specific Protocol Architecture for Wireless Microsensor Networks, IEEE Transactions on Wireless Communications, vol. 1, no.

23. 4, pp. 660670, 2002.

24. [19] Lindsey S. and Raghavendra C., PEGASIS: Power-Efficient Gathering in Sensor Information Systems, In Proc. IEEE Aerospace Conference, USA, Montana, 2002, Vol. 3, pp. 1125-1130

25. [20] Manjeshwar A. and Agrawal D. P., "TEEN: a routing protocol for enhanced efficiency in wireless sensor networks," Proceedings 15th International Parallel and Distributed Processing Symposium. IPDPS

26. 2001, San Francisco, CA, USA, 2001, pp. 2009-2015.

27. [21] Li Q.; Aslam J. and Rus D., Hierarchical Power-aware Routing in Sensor Networks, In Proc. DIMACS Workshop on Pervasive Networking, California, 2001, pp. 25-27

28. [22] Younis O. and Fahmy S., "HEED: a hybrid, energy-efficient, distributed clustering approach for ad hoc sensor networks," in IEEE Transactions on Mobile Computing, vol. 3, no. 4, pp. 366-379, Oct.-Dec. 2004. 